\title{
THE POWERS OF A MAXIMAL IDEAL IN A BANACH ALGEBRA AND ANALYTIC STRUCTURE
}

BY

\author{
T. T. READ( $\left.{ }^{1}\right)$
}

\begin{abstract}
Sufficient conditions are given for the existence of an analytic variety at an element $\phi$ of the spectrum of a commutative Banach algebra with identity. An associated graded algebra first considered by S. J. Sidney is used to determine the dimension of the analytic variety in terms of the closed powers of the maximal ideal which is the kernel of $\phi$.
\end{abstract}

1. Let $B$ be a complex commutative Banach algebra with identity. We shall denote the spectrum of $B$, the space of all multiplicative linear functionals on $B$, by $\Sigma(B)$. If $\phi$ is an element of $\Sigma(B)$, we denote by $B_{\phi}$ the maximal ideal which is the kernel of $\phi$. We shall be concerned with finding properties of $B_{\phi}$ which are sufficient for the existence of an analytic variety in $\Sigma(B)$ at $\phi$ and with studying the variety thus obtained. By an analytic variety at $\phi$ we mean the image of a one-to-one continuous mapping $F$ of a subvariety $V$ containing 0 of a domain in some $C^{r}$ into $\Sigma(B)$ such that $F(0)=\phi$ and such that for each $b \in B, \hat{b} \circ F$ is holomorphic on $V$.

Our investigations center on the sequence of powers $B_{\phi}^{n}$ of $B_{\phi}$, that is on the ideals generated by $n$-fold products of elements of $B_{\phi}$ and on the sequence of Banach spaces $B_{\phi} /\left(B_{\phi}^{2}\right)^{-},\left(B_{\phi}^{2}\right)^{-} /\left(B_{\phi}^{3}\right)^{-}, \ldots$ when the first of these is finite dimensional. In $\S 2$ we obtain a general condition sufficient for the existence of an analytic variety in $\Sigma(B)$ at $\phi$ (Theorem 2.5 ). We then study this variety more closely, obtaining its dimension at 0 in terms of the sequence of dimensions of the spaces $\left(B_{\phi}^{n}\right)^{-} /\left(B_{\phi}^{n+1}\right)^{-}$ (Theorem 3.5). We also show that the variety obtained from Theorem 2.5 is the largest which can be embedded in $\Sigma(B)$ at $\phi$ in the sense of having a maximal germ at 0 .

In $\$ 4$ we apply the general criterion of Theorem 2.5 to show that if the linear space $B_{\phi} / B_{\phi}^{2}$ is finite dimensional, then there is an analytic variety in $\Sigma(B)$ at $\phi$ which is a neighborhood of $\phi$ in the metric topology for $\Sigma(B)$. This result has also been obtained by Andrew Browder [1]. It follows from Theorem 3.5 that in this situation the variety is nontrivial if and only if $\left(B_{\phi}^{n}\right)^{-} \neq\left(B_{\phi}^{n+1}\right)^{-}$for all $n$.

Presented in part to the Society, January 25, 1968 under the title Analytic structure in the spectrum of a Banach algebra; received by the editors March 10, 1970 and, in revised form, February 4, 1971.

AMS 1970 subject classifications. Primary 46J20; Secondary 23B10, 46J10.

Key words and phrases. Commutative Banach algebra with identity, powers of a maximal ideal, analytic structure, dimension of an analytic variety, graded algebra.

( $\left.{ }^{1}\right)$ Research partially supported by a National Science Foundation Graduate Fellowship. 
Gleason [4] proved that if $B_{\phi}$ is algebraically finitely generated, that is if there are elements $g_{1}, \ldots, g_{t} \in B_{\phi}$ such that $B_{\phi}=B g_{1}+\cdots+B g_{t}$, then there is an analytic variety at $\phi$ which is open in the Gelfand topology for $\Sigma(B)$. It is easy to see that if $B_{\phi}$ is generated in the above sense by $g_{1}, \ldots, g_{t}$, then $\operatorname{dim}\left(B_{\phi} / B_{\phi}^{2}\right) \leqq t$, so that Theorem 4.1 is a generalization of Gleason's result. Indeed the hypothesis of Theorem 4.1 is strictly weaker, since it is not in general true that varieties obtained from Theorem 4.1 are open in the Gelfand topology (Example 5.1).

On the other hand, it is possible for $\phi$ to be an element of an analytic disc which is open in the Gelfand topology and yet have $\operatorname{dim}\left(B_{\phi} /\left(B_{\phi}^{2}\right)^{-}\right)$fail to be finite (Example 5.3). Thus the sufficient conditions so far obtained are far from being necessary, even for open analytic structure.

The material of this paper, except for $\S 4$, is essentially contained in the author's doctoral dissertation presented to Yale University. The author wishes to thank Professor E. L. Stout for much helpful advice and patient encouragement in the preparation of this dissertation.

2. In this section we develop our approach to finding varieties and prove a theorem giving sufficient conditions for the existence of a variety (Theorem 2.5). We begin with a simple observation. Here $\mathcal{O}[V]$ denotes the algebra of functions holomorphic on $V$, a subvariety of a domain in some $C^{r}$.

Proposition 2.1. Let $B$ be a commutative Banach algebra with identity, and let $\Phi: B \rightarrow \mathcal{O}[V]$ be a homomorphism whose range separates the points of $V$. Then the dual mapping $\Phi^{*}: V \rightarrow \Sigma(B)$ is an analytic variety in $\Sigma(B)$. If the range of $\Phi$ is dense in $\mathcal{O}[V]$ in the topology of uniform convergence on compact subsets of $V$, then $\Phi^{*}$ is a homeomorphism. Conversely, if $F: V \rightarrow \Sigma(B)$ is an analytic variety, then there is a homomorphism $\Phi: B \rightarrow \mathcal{O}[V]$ such that $\Phi^{*}=F$.

We remark that $\Phi[B]$ is contained in the algebra of bounded holomorphic functions on $V$. In fact, $\|\Phi(b)\|_{V} \leqq\|b\|$ since for any $z \in V, b \rightarrow \Phi(b)(z)$ is a multiplicative linear functional.

The homomorphisms which we shall now construct will carry a subset $\left\{w_{1}, \ldots, w_{r}\right\}$ of $B_{\phi}$ onto the coordinate functions in $C^{r}$. Thus $\Phi$ will have dense range and $\Phi^{*}$ will be the inverse of a restriction of the canonical map of $\Sigma(B)$ onto the joint spectrum of $w_{1}, \ldots, w_{r}$.

Let $\phi$ be an element of $\Sigma(B)$. We shall suppose from now on that $\operatorname{dim}\left(B_{\phi} /\left(B_{\phi}\right)^{-}\right)$ $=r$ is finite. The following proposition demonstrates that for finitely generated Banach algebras this condition is automatically satisfied.

Proposition 2.2. Let $B$ be a commutative Banach algebra with identity. If polynomials in 1 and $b_{1}, \ldots, b_{r}$ are dense in $B$, then for each $\phi \in \Sigma(B)$, the classes of $a_{i}$ $=b_{i}-\hat{b}_{i}(\phi)$ span $B_{\phi} /\left(B_{\phi}^{2}\right)^{-}$. Consequently $\operatorname{dim}\left(B_{\phi} /\left(B_{\phi}^{2}\right)^{-}\right) \leqq r$ for all $\phi \in \Sigma(B)$.

Proof. Let $b \in B_{\phi}$ and $\varepsilon>0$ be given. There is a polynomial $P$ in $r$ indeterminates with no constant term such that $\left\|b-P\left(a_{1}, \ldots, a_{r}\right)\right\|<\varepsilon$. Hence $\left\|b-P+\left(B_{\phi}^{2}\right)^{-}\right\|_{q}<\varepsilon$, 
where $\|\cdot\|_{q}$ denotes the quotient norm in $B_{\phi} /\left(B_{\phi}^{2}\right)^{-}$. Thus the span of $a_{1}+\left(B_{\phi}^{2}\right)^{-}, \ldots, a_{r}+\left(B_{\phi}^{2}\right)^{-}$is dense in $B_{\phi} /\left(B_{\phi}^{2}\right)^{-}$. Since the span is finite dimensional, it is also closed and must exhaust $B_{\phi} /\left(B_{\phi}^{2}\right)^{-}$.

The direct sum $\sum_{n=0}^{\infty} \oplus\left(\left(B_{\phi}^{n}\right)^{-} /\left(B_{\phi}^{n+1}\right)^{-}\right)$is a graded linear space if the elements of $\left(B_{\phi}^{n}\right)^{-} /\left(B_{\phi}^{n+1}\right)^{-}$are taken as the homogeneous elements of degree $n$. Here we take $B_{\phi}^{0}=B$. Following [10] we define a multiplication with respect to which this sum becomes a graded algebra. (Consult [11] for this and related matters.) Let $a$ and $b$ be representatives of elements $[a]$ of $\left(B_{\phi}^{p}\right)^{-} /\left(B_{\phi}^{p+1}\right)^{-}$and $[b]$ of $\left(B_{\phi}^{q}\right)^{-} /\left(B_{\phi}^{q+1}\right)^{-}$ respectively. Thus $a \in\left(B_{\phi}^{p}\right)^{-}, b \in\left(B_{\phi}^{q}\right)^{-}$and $a b \in\left(B_{\phi}^{p+q}\right)^{-}$. The class of $a b$ $\bmod \left(B_{\phi}^{p+q+1}\right)^{-}$depends only on $[a]$ and $[b]$ and we define $[a][b]$ to be the element of $\left(B_{\phi}^{p+q}\right)^{-} /\left(B_{\phi}^{p+q+1}\right)^{-}$so determined.

If $\left\{W_{1}, \ldots, W_{r}\right\}$ is a basis for $B_{\phi} /\left(B_{\phi}^{2}\right)^{-}$, then there is a natural homogeneous homomorphism $\Lambda$ of degree 0 of the graded algebra $C\left[X_{\mathrm{i}}, \ldots, X_{r}\right]$ of polynomials in $r$ indeterminates into $\sum_{n=0}^{\infty} \oplus\left(\left(B_{\phi}^{n}\right)^{-} /\left(B_{\phi}^{n+1}\right)^{-}\right)$such that $\Lambda\left(X_{i_{1}}^{v_{1}} \cdots X_{i_{m}}^{v_{m}}\right)$ $=W_{i_{1}}^{v_{1}} \cdots W_{i_{m}}^{v_{m}}$ for all monomials in the $X$ 's and $W$ 's. The following result [10, Theorem 1] is basic to our construction.

THEOREM 2.3. The homomorphism $\Lambda$ maps $C\left[X_{1}, \ldots, X_{r}\right]$ onto

$$
\sum_{n=0}^{\infty} \oplus\left(\left(B_{\phi}^{n}\right)^{-} /\left(B_{\phi}^{n+1}\right)^{-}\right)
$$

It is easily verified that for each $n$, the number of monomials of degree $n$ in $r$ indeterminates is the binomial coefficient $C_{n+r-1, n}$. Thus for each $n$, $\operatorname{dim}\left(\left(B_{\phi}^{n}\right)^{-} /\left(B_{\phi}^{n+1}\right)^{-}\right) \leqq C_{n+r-1, n}$ and the homogeneous monomials of degree $n$ in $W_{1}, \ldots, W_{r} \operatorname{span}\left(B_{\phi}^{n}\right)^{-} /\left(B_{\phi}^{n+1}\right)^{-}$.

We remark that $\sum_{n=0}^{\infty} \oplus\left(B_{\phi}^{n} / B_{\phi}^{n+1}\right)$ may be given a similar graded algebra structure and that the analog of Theorem 2.3 holds if $\operatorname{dim}\left(B_{\phi} / B_{\phi}^{2}\right)$ is finite. In particular, $\operatorname{dim}\left(B_{\phi} / B_{\phi}^{n}\right)$ is then finite for each $n$.

Suppose that $w_{1}, \ldots, w_{r} \in B_{\phi}$ are representatives of a basis for $B_{\phi} /\left(B_{\phi}^{2}\right)^{-}$. We shall now associate with each $b \in B$ polynomials in $w_{1}, \ldots, w_{r}$. Such a polynomial has the form $\sum_{|i| \leqq n} \beta_{(i)} w^{(i)}$ where $(i)=\left(i_{1}, \ldots, i_{r}\right), w^{(i)}=w_{1}^{i_{1}} \cdots w_{r}^{i_{r}}$ and $|i|=\sum_{j=1}^{r} i_{j}$. We say that such a polynomial $p$ is a partial sum for $b$ of degree $n$ with respect to $\left\{w_{1}, \ldots, w_{r}\right\}$ if $b-p \in\left(B_{\phi}^{n+1}\right)^{-}$. We write $b \sim_{n} p$.

For each $b \in B$ and each $n$ there is at least one partial sum for $b$ of degree $n$. This is clear for $n=0$. If $p^{\prime}$ is a partial sum of degree $n-1$, then $b-p^{\prime} \in\left(B_{\phi}^{n}\right)^{-}$. Since the homogeneous monomials of degree $n$ in $w_{1}, \ldots, w_{r}$ are representatives of a set which spans $\left(B_{\phi}^{n}\right)^{-} /\left(B_{\phi}^{n+1}\right)^{-}$, there is a homogeneous polynomial $p_{n}$ of degree $n$ such that $b-p^{\prime}-p_{n} \in\left(B_{\phi}^{n+1}\right)^{-}$. Thus $b \sim_{n} p^{\prime}+p_{n}$. Each element has a unique partial sum of degree $n$ for each positive integer $n$ if and only if for each $n$ the homogeneous monomials of degree $n$ in $w_{1}, \ldots, w_{r}$ are representatives of a basis for $\left(B_{\phi}^{n}\right)^{-} /\left(B_{\phi}^{n+1}\right)^{-}$. By the remark following Theorem 2.3 , this occurs if and only if $\operatorname{dim}\left(\left(B_{\phi}^{n}\right)^{-} /\left(B_{\phi}^{n+1}\right)^{-}\right)=C_{n+r-1, n}$ for all $n$.

This situation arises for the uniform closure of the polynomials on the closed 
unit polydisc in $C^{r}$. If $\phi$ is the origin and $w_{1}, \ldots, w_{r}$ are the coordinate functions, then $f \sim_{n} p$ if and only if $p$ is a partial sum of the Taylor series for $f$ about 0 .

We collect a few simple properties of the correspondence $b \sim_{n} p$.

Proposition 2.4. Suppose that $w_{1}, \ldots, w_{r}$ are representatives of a basis for $B_{\phi} /\left(B_{\phi}^{2}\right)^{-}$. If $a, b \in B_{\phi}, \lambda \in C$ and $a \sim_{n} p, b \sim_{n} q$, then

(1) $a+b \sim_{n} p+q$,

(2) $\lambda a \sim_{n} \lambda p$,

(3) $a b \sim_{n} p q$.

We are now ready to state a first set of conditions sufficient for analytic structure.

THEOREM 2.5. Let $B$ be a commutative Banach algebra with identity and let $\phi$ be an element of $\Sigma(B)$ such that

(1) for some positive integer $r, \operatorname{dim}\left(B_{\phi} /\left(B_{\phi}^{2}\right)^{-}\right)=r$, and

(2) there are representatives $\left\{w_{1}, \ldots, w_{r}\right\}$ of a basis for $B_{\phi} /\left(B_{\phi}^{2}\right)^{-}$and a positive number $M$ such that for each element $b$ of $B$ there is a constant $C(b)$ and, for each $n, a$ partial sum $\sum_{|i| \leqq n} \beta_{(i)} w^{(i)}$ for $b$ of degree $n$ such that $\left|\beta_{(i)}\right| \leqq C(b) M^{|i|}$ for each $(i)$ with $|i| \leqq n$.

Then there is a subvariety $V$ of the polydisc $\Delta=\left\{z \in C^{r}:\left|z_{i}\right|<1 / M, i=1, \ldots, r\right\}$ and a homeomorphism $\Phi^{*}$ of $V$ into $\Sigma(B)$ such that $\Phi^{*}(0)=\phi$ and for each $b \in B$, $\hat{b} \circ \Phi^{*}$ is holomorphic on $V$.

It is convenient to set down at this point a lemma which will be useful in the identification and study of the variety $V$. We denote the ring of germs of holomorphic functions at the origin in $\boldsymbol{C}^{r}$ by ${ }_{r} \mathcal{O}$. If $f$ is holomorphic in a neighborhood of the origin, we denote the germ of $f$ at the origin by $f$.

LEMMA 2.6. For each $n$, the subset $\mathscr{J}_{n}$ of elements $\sum \beta_{(i)} z^{(i)}$ of ${ }_{r} \mathcal{O}$ such that $\sum_{|i| \leqq n} \beta_{(i,}, w^{(i)} \in\left(B_{\phi}^{n+1}\right)^{-}$is an ideal in ${ }_{r} \mathcal{O}$.

Proof. Since $\left(B_{\phi}^{n+1}\right)^{-}$is a linear subspace of $B, \mathscr{J}_{n}$ is a linear subspace of ${ }_{r} \mathcal{O}$. If $f=\sum \beta_{(i)} z^{(i)} \in \mathscr{J}_{n}$ and if $(k)$ is any multi-index, then the sum of the terms of degree at most $n$ in the power series expansion of $z^{(k)} f$ is $z^{(k)} \sum_{|i| \leqq n-|k|} \beta_{(i)} z^{(i)}$. But $w^{(k)} \sum_{|i| \leqq n-|k|} \beta_{(i)} w^{(i)}$ is the difference of $w^{(k)} \sum_{|i| \leqq n} \beta_{(i)} w^{(i)}$ and $w^{(k)} \sum_{n-|k|<|i| \leqq n} \beta_{(i)} w^{(i)}$, both of which are in $\left(B_{\phi}^{n+1}\right)^{-}$. Hence $z^{(k)} f \in \mathscr{J}_{n}$. By linearity $\boldsymbol{p} f \in \mathscr{J}_{n}$ whenever $f \in \mathscr{J}_{n}$ and $\boldsymbol{p}$ is a polynomial in ${ }_{r} \mathcal{O}$. Finally, let $g=\sum \gamma_{(i)} z^{(i)} \in{ }_{+} \mathcal{O}$. Then for any $f \in \mathscr{J}_{n}$, the terms of degree at most $n$ in the power series expansion of $f \boldsymbol{g}$ are the same as those of $f \sum_{|i| \leqq n} \gamma_{(i)} z^{(i)}$. But from the previous remark, $f \sum_{|i| \leqq n} \gamma_{(i)} z^{(i)} \in \mathscr{J}_{n}$. It follows that $\mathscr{J}_{n}$ is an ideal in ${ }_{r} \mathcal{O}$.

COROLlaRY 2.7. $\mathscr{J}=\left\{\sum \beta_{(i)} z^{(i)} \in{ }_{r} \mathcal{O}: \sum_{|i| \leqq n} \beta_{(i)} w^{(i)} \in\left(B_{\phi}^{n+1}\right)^{-}\right.$for each $\left.n\right\}$ is an ideal in, $\mathcal{O}$.

Proof of Theorem 2.5. Fix $b \in B$. Let $\left\{P_{n}\right\}$ be a sequence of polynomials in $r$ indeterminates such that, for each $n, p_{n}=P_{n}\left(w_{1}, \ldots, w_{r}\right)$ is a partial sum for $b$ of 
degree $n$ satisfying (2). On any closed polydisc $D(\gamma)=\left\{z:\left|z_{i}\right| \leqq \gamma, i=1, \ldots, r\right\}$ with $\gamma<1 / M$ the sequence of polynomials $f_{n}=P_{n}\left\{z_{1}, \ldots, z_{r}\right\}$ in $r$ complex variables satisfies $\left|f_{n}(z)\right| \leqq C(b) \sum_{|i| \leqq n}(M \gamma)^{|i|} \leqq C \sum_{n=0}^{\infty} K^{n}$ for $M \gamma<K<1$ and some $C>0$. Hence a subsequence of $\left\{f_{n}\right\}$ converges uniformly on compact subsets of $\Delta$ to a holomorphic function $f$. We may thus define a mapping $\Psi$ of $B$ into $\mathcal{O}[\Delta]$ by $\Psi(b)$ $=f$.

Now let $I=\{f \in \mathcal{O}[\Delta]: f \in \mathscr{J}\}$, and let $V$ be the subvariety of $\Delta$ of common zeros of elements of $I$. We assert that $V$ is the variety we seek, that is, that the map $\Phi: B \rightarrow \mathcal{O}[V]$ obtained by setting $\Phi(b)=\Psi(b) \mid V$ is a homomorphism.

We show that $\Phi$ is multiplicative. Suppose $a, b \in B, \Psi(a)=f, \Psi(b)=g, \Psi(a b)=h$ where $f$ (resp. $g, h$ ) is obtained as the limit of a sequence of polynomials $f_{n}$ (resp. $g_{n}$, $\left.h_{n}\right)=P_{n}\left(z_{1}, \ldots, z_{r}\right)$ where $p_{n}$ (resp. $\left.q_{n}, r_{n}\right)=P_{n}\left(w_{1}, \ldots, w_{r}\right) \sim_{n} a$ (resp. $\left.b, a b\right)$. It is easy to see that $p_{i} q_{j}-r_{k} \in\left(B_{\phi}^{m+1}\right)^{-}$whenever $m \leqq \min \{i, j, k\}$. Hence $\boldsymbol{f}_{i} \boldsymbol{g}_{j}-\boldsymbol{h}_{\boldsymbol{k}} \in \mathscr{J}_{m}$. It follows from the Closure of Modules Theorem [6, Theorem IID3] that $\boldsymbol{f} \boldsymbol{g}-\boldsymbol{h} \in \mathscr{J}$. But this implies $f g-h \in I$, that is, $\Phi(a) \Phi(b)=f g|V=h| V=\Phi(a b)$. A similar argument gives that $\Phi$ is linear and the proof is complete.

We remark that although $f=\Psi(b)$ depends in general on the choice of the sequence $\left\{P_{n}\right\}, \Phi$ is independent of this choice. Indeed if also $q_{n}=Q_{n}\left(w_{1}, \ldots, w_{r}\right)$ is a sequence of partial sums for satisfying (2), and if a subsequence of $g_{n}$ $=Q_{n}\left(z_{1}, \ldots, z_{r}\right)$ converges to $g$, then an argument similar to the above leads to $\boldsymbol{f}-\boldsymbol{g} \in \mathscr{J}$.

On the other hand, it is easy to see that for $f=\Psi(b)=\sum \beta_{(i)} z^{(i)}$ we have for each $n$, $\boldsymbol{f}-\boldsymbol{f}_{n} \in \mathscr{J}_{n}$. Hence $b \sim_{n} \sum_{|i| \leqq n} \beta_{(i)} w^{(i)}$ for each $n$. We shall make use of this remark in the proof of Proposition 2.8 and again in $\$ 4$.

The nature of $V$ is not yet at all clear. In fact, it is not even clear whether $V$ contains any points other than 0 . We shall see in the next section that the dimension of $V$ at 0 is related to the structure of $B_{\phi}$. In particular, it is positive if $\left(B_{\phi}^{n}\right)^{-}$ $\neq\left(B_{\Phi}^{n+1}\right)^{-}$for all $n$.

We note that (2) in the hypotheses of Theorem 2.5 cannot be omitted. Indeed, Sidney [10, Example 5.18] has constructed a Swiss cheese algebra whose spectrum contains an element $\phi$ such that $\operatorname{dim}\left(\left(B_{\phi}^{n}\right)^{-} /\left(B_{\phi}^{n+1}\right)^{-}\right)=1$ for each $n$. We show next that at least (2) does not depend on the choice of $\left\{w_{1}, \ldots, w_{r}\right\}$.

Proposition 2.8. Let $\left\{W_{1}, \ldots, W_{r}\right\}$ and $\left\{Y_{1}, \ldots, Y_{r}\right\}$ be bases for $B_{\phi} /\left(B_{\phi}^{2}\right)^{-}$and let $\left\{w_{1}, \ldots, w_{r}\right\}$ and $\left\{y_{1}, \ldots, y_{r}\right\}$ be representatives. If $(2)$ holds with respect to $\left\{w_{1}, \ldots w_{r}\right\}$ then (2) also holds with respect to $\left\{y_{1}, \ldots, y_{r}\right\}$.

Proof. Let $\Psi$ denote the mapping of $B$ into $\mathcal{O}[\Delta]$ constructed as in the proof of Theorem 2.5 with respect to $\left\{w_{1}, \ldots, w_{r}\right\}$. For $k=1, \ldots, r$ we have $\Psi\left(y_{k}\right)(z)$ $=\sum \delta_{k,(i)} z^{(i)}$. Then the matrix $\left(\delta_{k,(i)}\right), k=1, \ldots, r,|i|=1$, is nonsingular. Thus the Jacobian matrix of the holomorphic mapping $F\left(z_{1}, \ldots, z_{r}\right)=\left(\Psi\left(y_{1}\right)(z), \ldots, \Psi\left(y_{r}\right)(z)\right)$ is nonsingular in a neighborhood of the origin in $C^{r}$. We have then an inverse map 
$G$ defined in a neighborhood $\Delta^{\prime}$ of the origin in $C^{r}$ [6, Theorem IB7], and for each $b \in B$ the function $\Psi^{\prime}(b)(\zeta)=\Psi(b)(G(\zeta))$ is holomorphic in $\Delta^{\prime}$.

We assert that if $\Psi^{\prime}(b)$ has the power series expansion $\sum \alpha_{(i)} \zeta^{(i)}$, then for each $n$ $\sum_{|i| \leqq n} \alpha_{(i)} y^{(i)}$ is a partial sum of degree $n$ for $b$ with respect to $\left\{y_{1}, \ldots, y_{r}\right\}$. We have that $y_{k} \sim_{n} \sum_{|i| \leqq n} \delta_{k,(i)} w^{(i)}$ with respect to $\left\{w_{1}, \ldots, w_{r}\right\}$ for $k=1, \ldots, r$ so that substitution of these polynomials into $\sum_{|i| \leqq n} \alpha_{(i)} y^{(i)}$ yields a polynomial $p$ of degree $n$ in $w_{1}, \ldots, w_{r}$ such that $p-\sum_{|i| \leqq n} \alpha_{(i)} y^{(i)} \in\left(B_{\phi}^{n+1}\right)^{-}$. On the other hand, the coefficients of $p$ are the same as those of degree at most $n$ in the power series expansion of $\Psi^{\prime}(b)(F(z))=\Psi(b)(z)$. Thus, by the remark following the proof of Theorem 2.5, $b-p \in\left(B_{\phi}^{n+1}\right)^{-}$. It follows that $b-\sum_{|i| \leqq n} \alpha_{(i)} y^{(i)} \in\left(B_{\phi}^{n+1}\right)^{-}$as asserted. It is clear that for some $M_{1},\left|\alpha_{(i)}\right| \leqq C^{\prime}(b) M_{1}^{|i|}$ for all $(i)$ and all $b \in B$. Thus (2) is also satisfied for these partial sums with respect to $\left\{y_{1}, \ldots, y_{r}\right\}$.

3. Our principal objective in this section is to relate the dimension at the origin of the variety $V$ obtained in Theorem 2.5 to the structure of $B_{\phi}$. We shall also show that no variety with a larger germ at the origin can be embedded in $\Sigma(B)$ at $\phi$. This notion will be made more precise later.

We begin by stating two theorems on graded algebras [11, Chapter VII, Theorems 41 and 42]. For these theorems and the rest of the section we assign to the zero polynomial the degree -1 .

THEOREM 3.1 (HILBERT-SERRE). Let $R=\sum \oplus R_{n}$ be a graded algebra which is the homomorphic image of a polynomial algebra $C\left[X_{1}, \ldots, X_{r}\right]$ modulo a homogeneous ideal I. Then there is a polynomial $\pi$ of degree at most $r-1$ such that $\pi(n)=\operatorname{dim} R_{n}$ for all large $n$.

THEOREM 3.2. If the degree of the polynomial $\pi$ is $s-1$, then $s$ is the greatest transcendence degree over $C$ of the quotient rings $C\left[X_{1}, \ldots, X_{r}\right] / P$ where $P$ is an isolated prime ideal of $I$.

By Theorem 2.3 if $\phi \in \Sigma(B)$ is such that $\operatorname{dim}\left(B_{\phi} /\left(B_{\phi}^{2}\right)^{-}\right)=r$, and if the set $\left\{W_{1}, \ldots, W_{r}\right\}$ is a basis for $B_{\phi} /\left(B_{\phi}^{2}\right)^{-}$, then there is a natural homogeneous homomorphism of $C\left[X_{1}, \ldots, X_{r}\right]$ onto $\sum_{n=0}^{\infty} \oplus\left(\left(B_{\phi}^{n}\right)^{-} /\left(B_{\phi}^{n+1}\right)^{-}\right)$. Thus there is a polynomial $\pi$ of degree at most $r-1$ such that $\pi(n)=\operatorname{dim}\left(\left(B_{\phi}^{n}\right)^{-} /\left(B_{\phi}^{n+1}\right)^{-}\right)$for all large $n$. We have also the following result.

COROLlaRY 3.3. If $\left\{W_{1}, \ldots, W_{r}\right\}$ is a basis for $B_{\phi} /\left(B_{\phi}^{2}\right)^{-}$then there are selements of this set (which we may take to be $W_{1}, \ldots, W_{s}$ ) such that for each $n$ the set of homogeneous monomials of degree $n$ in $W_{1}, \ldots, W_{s}$ is linearly independent in $\left(B_{\phi}^{n}\right)^{-} /\left(B_{\phi}^{n+1}\right)^{-}$. It is not possible to find $s+1$ elements of this set with this property.

Proof. Let $P$ be an isolated prime ideal of $I$ such that the transcendence degree of $C\left[X_{1}, \ldots, X_{r}\right] / P=C\left[Y_{1}, \ldots, Y_{r}\right]$ (where $Y_{i}$ is the $P$ residue of $X_{i}$ ) is $s$. We may assume that $\left\{Y_{1}, \ldots, Y_{s}\right\}$ is a transcendence basis of $C\left[Y_{1}, \ldots, Y_{r}\right]$ over $C$. Since $I \subset P$ there is a natural homogeneous homomorphism of $\sum_{n=0}^{\infty} \oplus\left(\left(B_{\phi}^{n}\right)^{-} /\left(B_{\phi}^{n+1}\right)^{-}\right)$ 
onto $C\left[Y_{1}, \ldots, Y_{r}\right]$ such that $W_{i}$ is carried onto $Y_{i}, i=1, \ldots, r$. Now it is clear that $W_{1}, \ldots, W_{s}$ have the desired property, since any algebraic relation among $W_{1}, \ldots, W_{s}$ would induce the same relation among $Y_{1}, \ldots, Y_{s}$. The last assertion follows from the fact that if $W_{1}, \ldots, W_{s+1}$ were as in the conclusion, then $\operatorname{dim}\left(\left(B_{\phi}^{n}\right)^{-} /\left(B_{\phi}^{n+1}\right)^{-}\right) \geqq C_{n+s, n}$, a polynomial of degree $s$ as a function of $n$.

Theorems 3.1 and 3.2 are also relevant to the dimension of a germ of a variety in $C^{r}$. If $s<r$ we may consider ${ }_{s} \mathcal{O}$ as the subring of ${ }_{r} \mathcal{O}$ of "functions depending on only the first $s$ variables." Then the dimension of the prime ideal $\mathscr{P}$ in ${ }_{r} \mathcal{O}$ (and of the irreducible variety $V$ such that $\mathscr{P}$ is the ideal of the germ of $V$ at the origin) is the unique integer $s$ such that in a suitable coordinate system $\mathscr{P} \cap{ }_{s} \mathcal{O}=(0)$ and ${ }_{r} \mathcal{O} / \mathscr{P}$ is integral over ${ }_{s} \mathcal{O}[6$, Chapter III $]$.

If $\mathscr{I}$ is an ideal in ${ }_{r} \mathcal{O}$ and if $\mathscr{M}_{\mathscr{G}}$ is the maximal ideal in ${ }_{r} \mathcal{O} / \mathscr{I}$, then $\sum_{n=0}^{\infty} \oplus\left(\mathscr{M}_{\mathscr{S}}^{n} / \mathscr{M}_{\mathscr{S}}^{n+1}\right)$ may be given a graded algebra structure in the same manner as $\sum_{n=0}^{\infty} \oplus\left(\left(B_{\phi}^{n}\right)^{-} /\left(B_{\phi}^{n+1}\right)^{-}\right)$, and is the homomorphic image of $C\left[X_{1}, \ldots, X_{r}\right]$. Thus there is a polynomial $\pi_{\mathscr{I}} \operatorname{such}$ that $\pi_{\mathscr{G}}(n)=\operatorname{dim}\left(\mathscr{M}_{\mathscr{G}}^{n} / \mathscr{M}_{\mathscr{G}}^{n+1}\right)$ for all large $n$.

Proposition 3.4. Let $\mathscr{P}$ be a prime ideal in ${ }_{r} \mathcal{O}$. If the dimension of $\mathscr{P}$ is $s$, then the degree of $\pi_{\mathscr{P}}$ is $s-1$.

Proof. It is clear that the degree of $\pi_{\mathscr{g}}$ is at most $s-1$, for otherwise as in the proof of Corollary 3.3 there would be $s^{\prime}>s$ such that $\mathscr{P} \cap{ }_{s^{\prime}} \mathcal{O}=(0)$. We prove the other inequality by induction. If the dimension of $\mathscr{P}$ is 1 , then we have $\mathscr{P} \cap{ }_{1} \mathcal{O}=(0)$ and we must show $\mathscr{M}_{\mathscr{P}}^{n} \neq \mathscr{M}_{\mathscr{P}}^{n+1}$ for all $n$. If for some $n, \mathscr{M}_{\mathscr{P}}^{n}=\mathscr{M}_{\mathscr{P}}^{n+1}$, then $z_{1}^{n}$ $\in \bigcap_{j=1}^{\infty}\left(\mathscr{P}+\mathscr{M}^{j}\right)$, that is, for each $j$ there is $f_{j} \in \mathscr{P}$ such that $f_{j}-z_{1}^{n}$ has total order at least $j$. Then $f_{j} \rightarrow z_{1}^{n}$ in the topology of simple convergence and it follows that $z_{1}^{n} \in \mathscr{P}[9$, Theorem 6.3.5], a contradiction.

Now suppose that the theorem has been proved for all $k<s$, that the dimension of $\mathscr{P}$ is $s$, and that the degree of $\pi_{\mathscr{P}}$ is $t-1<s-1$. If $I$ is the kernel of the homogeneous homomorphism of $\boldsymbol{C}\left[X_{1}, \ldots, X_{\mathrm{r}}\right]$ onto $\sum_{n=0}^{\infty} \oplus\left(\mathscr{M}_{\mathscr{P}}^{n} / \mathscr{M}_{\mathscr{P}}^{n+1}\right)$, then after suitable relabelling, no polynomial in $X_{1}, \ldots, X_{t}$ is in $I$. On the other hand, as in the proof of Corollary 3.3, for each $j>t I$ contains a polynomial in $X_{1}, \ldots, X_{t}, X_{j}$.

Let $\mathscr{I}$ be the ideal in ${ }_{r} \mathcal{O}$ generated by $\mathscr{P}$ and $z_{t}$, let $\mathscr{P}^{\prime}$ be a prime ideal associated with $\mathscr{I}$ and let $I^{\prime}$ be the kernel of the homogeneous homomorphism of $C\left[X_{1}, \ldots, X_{r}\right]$ onto $\sum_{n=0}^{\infty} \oplus\left(\mathscr{M}_{\mathscr{P}^{\prime}}^{n} / \mathscr{M}_{\mathscr{P}^{\prime}}^{n+1}\right)$. Then $I \subset I^{\prime}$ and $X_{t} \in I^{\prime}$. Hence for any prime ideal $P^{\prime} \supset I^{\prime}$, each $Y_{i}^{\prime}=X_{i}+P^{\prime}$ is algebraically dependent on $Y_{1}^{\prime}, \ldots, Y_{t-1}^{\prime}$. Hence the degree of the polynomial $\pi_{\mathscr{B}}$, is at most $t-2$. On the other hand, the dimension of $\mathscr{P}^{\prime}$ is $s-1$ [6, Theorem III C 14]. This contradiction completes the proof.

We are now ready to identity the dimension at the origin of the variety found in Theorem 2.5.

THEOREM 3.5. Let $B$ be a commutative Banach algebra with identity and let $\phi$ be an element of $\Sigma(B)$ which satisfies the hypotheses of Theorem 2.5. Let $s-1$ be the 
degree of the polynomial $\pi$ such that $\pi(n)=\operatorname{dim}\left(\left(B_{\phi}^{n}\right)^{-} /\left(B_{\phi}^{n+1}\right)^{-}\right)$for all large $n$. Then $s$ is the dimension at the origin of the variety $V$ constructed in the proof of Theorem 2.5.

Proof. Let $\mathscr{I}$ be the ideal in ${ }_{r} \mathcal{O}$ generated by the germs of functions in the ideal $I$ of Theorem 2.5. Then the variety of $\mathscr{I}$ is the germ of $V$ at the origin. It is clear from the definition of $I$ that $\mathscr{I}$ is contained in the ideal $\mathscr{J}$ of Corollary 2.7.

In order to show that the dimension of $V$ at the origin is at least $s$ it suffices to show that $\mathscr{I} \cap{ }_{s} \mathcal{O}=(0)$, for then $\mathscr{P} \cap{ }_{s} \mathcal{O}=(0)$ for some prime ideal $\mathscr{P}$ associated with $\mathscr{I}$. We shall in fact show that $\mathscr{J} \cap{ }_{s} \mathcal{O}=(0)$. Suppose $f=\sum \beta_{(i)} z^{(i)} \in \mathscr{J} \cap{ }_{s} \mathcal{O}$. Then $\sum_{|i|=1} \beta_{(i)} w^{(i)} \in\left(B_{\Phi}^{2}\right)^{-}$. But the set $\left\{w_{i}+\left(B_{\phi}^{2}\right)^{-}: i=1, \ldots, s\right\}$ is linearly independent. Hence $\beta_{(i)}=0$ for all $(i)$ such that $|i|=1$. By induction on $|i|$ and Corollary 3.3 it follows that $\beta_{(i)}=0$ for all $(i)$, and thus that $\mathscr{J} \cap{ }_{s} \mathcal{O}=(0)$ as asserted.

For the opposite inequality, let $\mathscr{P}$ be a prime ideal associated with $\mathscr{I}$ of maximum dimension. It suffices, in view of Proposition 3.4, to construct a homogeneous homomorphism of degree 0 of $\sum_{n=0}^{\infty} \oplus\left(\left(B_{\phi}^{n}\right)^{-} /\left(B_{\phi}^{n+1}\right)^{-}\right)$onto $\sum_{n=0}^{\infty} \oplus\left(\mathscr{M}_{\mathscr{P}}^{n} / \mathscr{M}_{\mathscr{P}}^{n+1}\right)$. The homomorphism $\Phi: B \rightarrow \mathcal{O}[V]$ induces a homomorphism $\Lambda: B \rightarrow{ }_{r} \mathcal{O} / \mathscr{P}$ such that $\Lambda\left[B_{\phi}\right] \subset \mathscr{M}_{\mathscr{P}}$. Thus $\Lambda\left[B_{\phi}^{n}\right] \subset \mathscr{M}_{\mathscr{P}}^{n}$. Once we show that $\Lambda\left[\left(B_{\phi}^{n}\right)^{-}\right] \subset \mathscr{M}_{\mathscr{P}}^{n}$, then $\Lambda$ will induce the desired homomorphism.

If $f \notin \mathscr{M}_{\mathscr{P}}^{n}$, then there is a linear functional $t$ on ${ }_{r} \mathcal{O} / \mathscr{P}$ such that $t$ annihilates $\mathscr{M}_{\mathscr{P}}^{n}$ while $t(f) \neq 0$. Thus to show $\Lambda\left[\left(B_{\phi}^{n}\right)^{-}\right] \subset \mathscr{M}_{\mathscr{P}}^{n}$ it suffices to show that any linear functional $t$ on ${ }_{r} \mathcal{O} / \mathscr{P}$ which annihilates some $\mathscr{M}_{\mathscr{P}}^{n}$ is continuous in the sense that if $f_{n} \rightarrow 0$ uniformly on some neighborhood $N$ of the origin on the variety of $\mathscr{P}$, then $t\left(f_{n}\right) \rightarrow 0$. To see this we remark first that $t$ induces a linear functional $\tau$ on, $\mathcal{O}$ by $\tau(\boldsymbol{g})=t(\boldsymbol{g}+\mathscr{P})$. Since $\tau$ annihilates $\mathscr{M}^{n}, \tau$ is a linear combination of partial derivatives at the origin of order less than $n$. Also, there is a neighborhood $W$ of the origin in $C^{r}$ and a constant $K$ such that each $f \in \mathcal{O}[N]$ extends to $F \in \mathcal{O}[W]$ with $\|F\|$ $\leqq K\|f\|\left[6\right.$, Corollary V B 4]. Hence a sequence $\left\{f_{n}\right\}$ converging uniformly to 0 on $N$ extends to a sequence $\left\{F_{n}\right\}$ converging uniformly to 0 on $W$. Then $t\left(f_{n}\right)=\tau\left(f_{n}\right)$ $\rightarrow 0$ and our assertion is established, concluding the proof.

It is an immediate consequence of the next result that the variety $V$ obtained in Theorem 2.5 is the largest analytic variety in $\Sigma(B)$ at $\phi$ in the sense that there is no analytic variety $V^{\prime}$ in $\Sigma(B)$ at $\phi$ whose germ at the origin properly contains the germ of $V$. We denote by $U$ the open unit disc in the complex plane.

THEOREM 3.6. Suppose that the hypotheses of Theorem 2.5 are satisfied for an element $\phi$ of $\Sigma(B)$ and that $V$ is the variety thus obtained. If $\theta^{*}: U \rightarrow \Sigma(B)$ is any analytic disc in $\Sigma(B)$ at $\phi$, then there is a neighborhood $U^{\prime} \subset U$ of the origin such that $\theta^{*}\left[U^{\prime}\right] \subset \Phi^{*}[V]$.

Proof. $\theta^{*}$ induces a homomorphism $\theta$ of $B$ into $\mathcal{O}[U]$ by $\theta(b)(z)=\hat{b}\left(\theta^{*}(z)\right)$. Let $\sigma: \Sigma(B) \rightarrow C^{r}$ denote the canonical map onto the joint spectrum of the elements 
$w_{1}, \ldots, w_{r}$ chosen as in Theorem 2.5. Let $U^{\prime}$ be a neighborhood of the origin such that $\sigma\left[\theta^{*}\left[U^{\prime}\right]\right] \subset \Delta$.

We assert that if $f \in \mathcal{O}[\Delta], f(z)=\sum \beta_{(i)} z^{(i)}$ and if, for each $n$ and some element $b$ of $B, b \sim_{n} p_{n}$ where $p_{n}=\sum_{|i| \leqq n} \beta_{(i)} w^{(i)}$, then

$$
\theta(b)(z)=f\left(\sigma\left(\theta^{*}(z)\right)\right) .
$$

For any $n, b-p_{n} \in\left(B_{\phi}^{n+1}\right)^{-}$. Hence $\theta\left(b-p_{n}\right)$ has a zero of order $n+1$ at the origin. Since $\theta\left(b-p_{n}\right)(z)=\theta(b)(z)-p_{n}\left(\sigma\left(\theta^{*}(z)\right)\right)$, this implies that the first $n$ coefficients of the Taylor series about zero of the two sides of $(+)$ agree and the assertion follows.

If we take $f$ to be an element of the ideal $I$ of the proof of Theorem 2.5 this gives, for any $z$ in $U^{\prime}, f\left(\sigma\left(\theta^{*}(z)\right)\right)=\theta(0)(z)=0$ and it follows that $\sigma\left[\theta^{*}\left[U^{\prime}\right]\right] \subset V$. Now for any $b \in B$ and $z \in U^{\prime}$, if we take $f=\Psi(b)$ and recall $\Psi(b) \mid V=\Phi(b)$ we have $\hat{b}\left(\theta^{*}(z)\right)$ $=\theta(b)(z)=\Phi(b)\left(\sigma\left(\theta^{*}(z)\right)\right)=\hat{b}\left(\Phi^{*}\left(\sigma\left(\theta^{*}(z)\right)\right)\right)$. Thus $\theta^{*}(z)=\Phi^{*}\left(\sigma\left(\theta^{*}(z)\right)\right)$ is in $\Phi^{*}[V]$.

It follows from Theorem 3.6 that an alternate definition of the variety $V$ in Theorem 2.5 would have led to the same result. For suppose in the situation of Theorem 2.5 that we define $V^{\prime}$ to be the subvariety of $\Delta$ consisting of the common zeros of all functions of the form $P\left(\Psi\left(b_{1}\right), \ldots, \Psi\left(b_{n}\right)\right)-\Psi\left(P\left(b_{1}, \ldots, b_{n}\right)\right)$ where $b_{1}, \ldots, b_{n} \in B$ and $P$ is a polynomial in $n$ indeterminates. Then $b \rightarrow \Psi(b) \mid V^{\prime}$ is a homomorphism so that $V^{\prime}$ is an analytic variety in $\Sigma(B)$ at $\phi$. It was essentially verified in the proof of Theorem 2.5 that each function of the above form is in $I$. Hence $V \subset V^{\prime}$. On the other hand, from Theorem 3.6 this inclusion cannot be proper. Thus $V=V^{\prime}$. The original definition, of course, had the advantage that the variety clearly does not depend on the choice of the functions $\Psi(b)$.

4. We shall now apply the results of the previous sections to prove

THEOREM 4.1. Let $B$ be a commutative Banach algebra with identity and let $\phi$ be an element of $\Sigma(B)$ such that $\operatorname{dim}\left(B_{\phi} / B_{\phi}^{2}\right)$ is finite. Then $\Sigma(B)$ contains an analytic variety at $\phi$ which is a neighborhood of $\phi$ in the metric topology. The variety is nontrivial if and only if $\left(B_{\phi}^{n}\right)^{-} \neq\left(B_{\phi}^{n+1}\right)^{-}$for all $n$.

Proof. We shall show that if $w_{1}, \ldots, w_{r}$ are representatives of a basis for $B_{\phi} /\left(B_{\phi}^{2}\right)^{-}$with $\left\|w_{i}\right\|=1$ for each $i$, then there are positive numbers $M$ and $C$ such that for each element $b$ of $B_{\phi}$ and for each $n$ there is a partial sum $p_{n}=\sum_{|i| \leqq n} \beta_{(i)} w^{(i)}$ for $b$ of degree $n$ such that

(1) $\left|\beta_{(i)}\right| \leqq C M^{|i|}\|b\|$ for each $(i)$ with $|i| \leqq n$,

(2) for all $\psi \in \Sigma(B)$ satisfying $\|\phi-\psi\| \leqq \frac{1}{2} D M$ we have $\left|\left(b-p_{n}\right)^{\wedge}(\psi)\right| \leqq C\left(\frac{1}{2}\right)^{n+1}\|b\|$. Here $D=\max (2 r, 16)$.

From (1) we will have that the hypotheses of Theorem 2.5 are satisfied and thus that there is an analytic variety in $\Sigma(B)$ at $\phi$. The last assertion of the theorem is then immediate from Theorem 3.5. From (2) we will have that for elements of the metric neighborhood $N=\left\{\psi:\|\phi-\psi\| \leqq \frac{1}{2} D M\right\}, \hat{b}(\psi)=\lim \hat{p}_{n}(\psi)=\lim f_{n}(\sigma(\psi))$ $=\Psi(b)(\sigma(\psi))$. Here $f_{n}(z)=\sum_{|i| \leqq n} \beta_{(i)} z^{(i)}$ and $\sigma$ is as before the map of $\Sigma(B)$ onto the 
joint spectrum of $w_{1}, \ldots, w_{r}$. Thus every function of the form $P\left(\Psi\left(b_{1}\right), \ldots, \Psi\left(b_{n}\right)\right)$ $-\Psi\left(P\left(b_{1}, \ldots, b_{n}\right)\right)$ vanishes on the set $\sigma[N]$. By the remark following Theorem 3.6, $\sigma[N] \subset V$. For any $\psi \in N, \Phi^{*}(\sigma(\psi))$ is the homomorphism $b \rightarrow \Phi(b)(\sigma(\psi))$ $=\hat{b}(\psi)$. Thus $N \subset \Phi^{*}[V]$ and we will have that $\Phi^{*}[V]$ is a metric neighborhood of $\phi$.

We now establish (1) and (2). First we note that for any integer $n$ it suffices to show that the assertion is true for all $b$ in a dense subset of $B$. For suppose the assertion true for each element of a sequence $\left\{b_{k}\right\}$ with $b_{k} \rightarrow b$. Let $p_{k}$ be the partial sum of degree $n$ for $b_{k}$. For any $(i)$ with $|i| \leqq n$, the $(i)$ th coefficients of the $p_{k}$ 's are uniformly bounded. Hence a subsequence of the $p_{k}$ 's converges to a polynomial $p$ of degree $n$ in $w_{1}, \ldots, w_{r}$. Since $b_{k}-p_{k} \in\left(B_{\phi}^{n+1}\right)^{-}$for each $k, b-p \in\left(B_{\phi}^{n+1}\right)^{-}$and it is clear that $p$ satisfies (1) and (2).

For each $b \in B_{\phi}, b=\sum_{|i|=1} \beta_{(i)} w^{(i)}+b_{2}, b_{2} \in\left(B_{\phi}^{2}\right)^{-}$, where the $\beta_{(i)}$ are uniquely determined. For each $(i)$ the linear functional $b \rightarrow \beta_{(i)}$ is continuous since it annihilates the closed subspace $\left(B_{\phi}^{2}\right)^{-}$of finite codimension in $B_{\phi}$. Hence there is a number $L$ such that $\left|\beta_{(i)}\right| \leqq L\|b\|$ for each $b$ and each $(i)$ with $|i|=1$. Since $\left\|w_{i}\right\|=1$, we have also $\left\|b_{2}\right\| \leqq(r L+1)\|b\|$.

We denote by $B_{\phi} \otimes_{\gamma} B_{\phi}$ the completion of the algebraic tensor product of $B_{\phi}$ with itself in the greatest cross norm. This is defined by $\left\|\sum a_{j} \otimes b_{j}\right\|=\inf \left\{\sum\left\|a_{j}\right\|\left\|b_{j}\right\|\right\}$ where the infimum is over all representations of the element of the algebraic tensor product [3]. Let $T: B_{\phi} \otimes_{\gamma} B_{\phi} \rightarrow B_{\phi}$ be the linear mapping which for elements of the algebraic tensor product is defined by $T\left(\sum a_{j} \otimes b_{j}\right)=\sum a_{j} b_{j}$. T is normdecreasing and the range of $T$ contains $B_{\phi}^{2}$. By assumption $B_{\Phi}^{2}$ has finite codimension in $B_{\phi}$. Thus the same is true of the range of $T$ and it follows that the range of $T$ is $\left(B_{\Phi}^{2}\right)^{-}[5$, Corollary IV.1.13].

Now by the Open Mapping Theorem there is $K>0$ such that for each $b \in\left(B_{\Phi}^{2}\right)^{-}$ there is an element $a$ with $T(a)=b$ and $\|a\| \leqq K\|b\|$. Moreover, for each $b$ in a dense subset of $\left(B_{\phi}^{2}\right)^{-}$we may assume that $a$ belongs to the algebraic tensor product, that is that $b=\sum a_{j} c_{j}$ with $a_{j}, c_{j} \in B_{\phi}$ and $\sum\left\|a_{j}\right\|\left\|c_{j}\right\| \leqq K\|b\|$.

We use this remark to expand certain elements $b$ of $B_{\phi}$. We have

$$
b=\sum_{|i|=1} \beta_{(i)} w^{(i)}+b_{2}, \quad b_{2} \in\left(B_{\Phi}^{2}\right)^{-} .
$$

For $b$ in a dense subset of $B_{\phi}$,

$$
\begin{aligned}
b & =\sum_{|i|=1} \beta_{(i)} w^{(i)}+\sum_{j} a_{j} c_{j}, \quad \sum\left\|a_{j}\right\|\left\|c_{j}\right\| \leqq K\left\|b_{2}\right\|, \\
& =\sum_{|i|=1} \beta_{(i)} w^{(i)}+\sum_{j}\left(\sum_{|i|=1} \alpha_{j,(i)} w^{(i)}+d_{j}\right)\left(\sum_{|i|=1} \gamma_{j,(i)} w^{(i)}+e_{j}\right),
\end{aligned}
$$

where $d_{j}, e_{j} \in\left(B_{\phi}^{3}\right)^{-}$. Thus

$$
b=\sum_{|i|=1} \beta_{(i)} w^{(i)}+\sum_{|i|=2}\left[\sum_{(0)<(k)<(i)}\left(\sum_{j} \alpha_{j,(k)} \gamma_{j,(i-k)}\right)\right] w^{(i)}+b_{3},
$$

where $b_{3} \in\left(B_{\Phi}^{2}\right)^{-}$. 
By induction, for any $n$ we may for a dense subset of $B_{\phi}$ iterate this process $n$ times at each step replacing each element of $\left(B_{\phi}^{2}\right)^{-}$in a sum such as (3) or (5) by a sum of products of elements of $B_{\phi}$, each represented as in (3). We may then collect terms as in (4) to obtain a representation

$$
b=\sum_{|i| \leqq n} \beta_{(i)} w^{(i)}+b_{n+1}, \quad b_{n+1} \in\left(B_{\phi}^{n+1}\right)^{-} .
$$

We assert that then $\left|\beta_{(i)}\right| \leqq C N_{(i)}[K L(r L+1)]^{|i|}\|b\|$ for each $(i)$ with $1 \leqq|i| \leqq n$. Here $C=1 / K(r L+1)$ and $N_{(i)}$ is the number of terms which must be collected to form $\beta_{(i)} w^{(i)}$. $N_{(i)}=1$ if $|i|=1$ and it will be seen below that $N_{(i)} \leqq(4 r)^{|i|}$ for all $(i)$. Thus we will have (1) with $M=4 r K L(r L+1)$.

If $|i|=1$, we have seen that $\left|\beta_{(i)}\right| \leqq L\|b\| \leqq C M\|b\|$. Now suppose that the assertion is true for $n-1$ and that for $b \in B_{\phi}$ the above process has been iterated $n$ times. Then it has been iterated $n-1$ times for each $a_{j}$ and $c_{j}$ appearing in (4). Thus

$$
a_{j}=\sum_{|1| \leqq n-1} \alpha_{j,(i)} w^{(i)}+d_{j, n}, \quad c_{j}=\sum_{|i| \leqq n-1} \gamma_{j,(i)} w^{(i)}+e_{j, n}
$$

with $d_{j, n}, e_{j, n} \in\left(B_{\phi}^{n}\right)^{-}$and

$$
\left|\alpha_{j,(i)}\right| \leqq C N_{(i)}[K L(r L+1)]^{|i|}\left\|a_{j}\right\|, \quad\left|\gamma_{j,(i)}\right| \leqq C N_{(i)}[K L(r L+1)]^{|i|}\left\|c_{j}\right\|
$$

for each $j$ and each $(i)$ with $1 \leqq|i| \leqq n-1$. Now, for $2 \leqq|i| \leqq n$,

$$
\beta_{(i)}=\sum_{(0)<(k)<(i)}\left(\sum_{j} \alpha_{j,(k)} \gamma_{j,(i-k)}\right)
$$

so

$$
\left|\beta_{(i)}\right| \leqq C^{2} N_{(i)}[K L(r L+1)]^{|i|} \sum_{j}\left\|a_{j}\right\|\left\|c_{j}\right\| \leqq C N_{(i)}[K L(r L+1)]^{|i|}\|b\|
$$

as asserted, where we note $N_{(i)}=\sum_{(0)<(k)<(i)} N_{(k)} N_{(i-k)}$.

We now determine the integers $N_{(i)}$. For $r=1$, that is in case $N_{i}=\sum_{k=1}^{i-1} N_{k} N_{i-k}$ it is well known that $N_{i}=(2 i-2) ! / i !(i-1)$ ! (see, for example, [7, p. 25]). In the general case a slight modification of the argument in the above reference yields $N_{(i)}=[(2|i|-2) ! /|i| !(|i|-1) !]\left[|i| ! / i_{1} ! \ldots i_{r} !\right]$. Since the first term in this product is no more than $4^{|i|}$ and the second no more than $r^{|i|}$, we obtain the generous estimate $N_{(i)} \leqq(4 r)^{|i|}$.

To complete the proof we verify that (2) also holds for all $b$ in a dense subset of $B_{\phi}$. We have $b=\sum_{|i|=1} \beta_{(i)} w^{(i)}+b_{2}, b_{2}=\sum a_{j} c_{j}$ as before. Now

$$
\begin{aligned}
\left|\hat{b}_{2}(\psi)\right| & \leqq \sum\left\|a_{j}\right\|\left\|c_{j}\right\|\|\phi-\psi\|^{2} \leqq(1 / C)\|\phi-\psi\|^{2}\|b\| \\
& \leqq C M^{2}\|\phi-\psi\|^{2}\|b\| \leqq C\left(\frac{1}{2}\right)^{2}\|b\|
\end{aligned}
$$

if $\|\phi-\psi\| \leqq \frac{1}{2} D M$. Thus (2) holds for $n=1$. Suppose that it holds for $n-1$. Then, returning to the computation for (1), we may assume in addition that $\left|\hat{d}_{j, n}(\psi)\right|$ $\leqq C\left(\frac{1}{2}\right)^{n}\left\|a_{j}\right\|,\left|\hat{e}_{j, n}(\psi)\right| \leqq C\left(\frac{1}{2}\right)^{n}\left\|c_{j}\right\|$ for all $\psi$ such that $\|\phi-\psi\| \leqq \frac{1}{2} D M$. Then

$$
b=\sum_{|i|=1} \beta_{(i)} w^{(i)}+\sum_{j} a_{j} c_{j}=\sum_{|i| \leqq n} \beta_{(i)} w^{(i)}+b_{n+1}
$$


where

$$
\begin{aligned}
b_{n+1}= & \sum_{n+1 \leqq|i| \leqq 2 n-2}\left[\sum_{(k)}\left(\sum_{j} \alpha_{j,(k)} \gamma_{j,(i-k)}\right)\right] w^{(i)} \\
& +\sum_{j}\left(d_{j, n} \sum_{1 \leqq|1| \leqq n-1} \gamma_{j,(i)} w^{(i)}\right) \\
& +\sum_{j}\left(e_{j, n} \sum_{1 \leqq|i| \leqq n-1} \alpha_{j,(i)} w^{(i)}\right)+\sum_{j} d_{j, n} e_{j, n} .
\end{aligned}
$$

Hence if $\|\phi-\psi\| \leqq \frac{1}{2} D M$,

$$
\begin{aligned}
\left|\hat{b}_{n+1}(\psi)\right| \leqq & \sum_{m=n+1}^{2 n-2}\left(C_{r+m-1, m} C M^{m}\|\phi-\psi\|^{m}\|b\|\right) \\
& +2\left(\frac{1}{2}\right)^{n}\|b\| \sum_{m=1}^{n-1}\left(C M^{m}\|\phi-\psi\|^{m}\right)+C\left(\frac{1}{2}\right)^{2 n}\|b\| \\
\leqq & C\left(\frac{1}{2}\right)^{n+1}\|b\|\left[\left(r^{n+1} / D^{n+1}\right) \sum_{m=0}^{n-3} r^{m}(1 / 2 D)^{m}+4 \sum_{m=1}^{n-1}(1 / 2 D)^{m}+\left(\frac{1}{2}\right)^{n-1}\right] \\
\leqq & C\left(\frac{1}{2}\right)^{n+1}\|b\|\left[\frac{1}{8}(1 /(1-r / 2 D))+(2 / D) /(1-1 / 2 D)+\frac{1}{2}\right] \\
\leqq & C\left(\frac{1}{2}\right)^{n+1}\|b\|\left[\frac{1}{4}+\frac{1}{4}+\frac{1}{2}\right]=C\left(\frac{1}{2}\right)^{n+1}\|b\| .
\end{aligned}
$$

Here we have used the inequality $C_{m+r-1, m} \leqq r^{m}$ valid for all positive integers $r$ and $m$. Thus (2) also holds and the proof of Theorem 4.1 is complete.

A point derivation on $B$ at $\phi$ is a linear functional on $B$ which annihilates the constants and $B_{\phi}^{2}$. A consequence of Theorem 4.1 is then

COROLlARY 4.2. If every point derivation at $\phi$ is bounded, then $\Sigma(B)$ contains an analytic variety at $\phi$ which is a neighborhood of $\phi$ in the metric topology.

Proof. By assumption $B_{\phi}^{2}=\left(B_{\phi}^{2}\right)^{-}$. Moreover, $\operatorname{dim}\left(B_{\phi} / B_{\phi}^{2}\right)$ is finite, for otherwise any unbounded linear functional on $B_{\phi} / B_{\phi}^{2}$ would induce an unbounded point derivation at $\phi$.

5. We consider now three uniform algebras. The first demonstrates that the analytic structure obtained from Theorem 4.1 need not be open in the Gelfand topology, and that $\phi$ may be an element of the Silov boundary.

EXAMPLE 5.1. In $C^{2}$ let $X$ be the union of the sets $D_{1}=\left\{\left(z_{1}, z_{2}\right):\left|z_{1}\right| \leqq 1, z_{2}=0\right\}$ and $D_{2}=\left\{\left(z_{1}, z_{2}\right): z_{1}=0,\left|z_{2}-1\right| \leqq 1\right\}$, and let $A$ be the closure of the polynomials on $X .(0,0)$ is in the Silov boundary of $A$. Let $A_{0}$ denote the maximal ideal of functions which vanish at $(0,0)$. If $f \in A_{0}$, then $f=f_{1}+f_{2}$ where $f_{1}, f_{2} \in A$ and $f_{1}\left|D_{2}=f_{2}\right| D_{1}=0$. Since $(0,0)$ is a peak point for $A \mid D_{2}, f_{2} \in \bigcap_{n=1}^{\infty} A_{0}^{n}[2$, Theorem 4.1]. Since $A \mid D_{1}$ is the disc algebra, $f_{1} \in A_{0}^{n}$ if and only if $f_{1}=z_{1}^{n} g, g \in A$. Hence $\operatorname{dim}\left(A_{0} / A_{0}^{2}\right)=1$ for all $n,\left\{z_{1}+A_{0}^{2}\right\}$ is a basis for $A_{0} / A_{0}^{2}$ and the function $\Phi^{*}$ of Theorem 2.5 maps the open unit disc $U$ onto $U \times\{0\}$.

The other two examples will be drawn from a class of uniform algebras studied by S. J. Sidney [10] whose relevant properties we first briefly describe. 
Let $I$ be an index set, let $Z=\prod\left\{D_{i}: i \in I\right\}$ be a product of closed unit discs, and let $T$ denote the product of circles which is its distinguished boundary. We denote by $P$ the closure on $Z$ of the polynomials in the coordinate functions and by $P_{0}$ the maximal ideal of functions which vanish at the origin. Let $B$ be a uniform algebra with Silov boundary $\Gamma$. Then we shall be interested in the uniform algebra $A$ which is the closure on $\Gamma \times Z$ of $C(\Gamma) \otimes P_{0}^{m}+B \otimes P$. Here $C(\Gamma)$ denotes the algebra of all complex valued continuous functions on $\Gamma$ and $\otimes$ denotes the algebraic tensor product. $\Sigma(A)$ may be identified with $(\Sigma(B) \times\{0\}) \cup(\Gamma \times Z)$ and the Silov boundary for $A$ is $\Gamma \times T$. If $\phi=(\psi, 0)$ for some $\psi \in \Sigma(B) \mid \Gamma$, then for each $n,\left(A_{\phi}^{n}\right)^{-}$is the closed linear span of $C(\Gamma) \otimes P_{0}^{m}$, and $B_{\psi}^{j} \otimes P_{0}^{n-j}$ for $j=0, \ldots, n$. (We recall $B_{\psi}^{0}=B$, $P_{0}^{0}=P$.)

EXAMPLE 5.2. It is possible for $\phi$ to be isolated, even though $\operatorname{dim}\left(A_{\phi} / A_{\phi}^{2}\right)=1$. In fact for any $k$ we may choose $A$ so that $\operatorname{dim}\left(A_{\phi}^{j} / A_{\phi}^{j+1}\right)=1$ for $j=1, \ldots, k-1$ but $A_{\phi}^{k}=A_{\phi}^{k+1}$. We choose $Z$ to be a single closed disc, take $m=k$, and let $B$ be the algebra of bounded analytic functions on the open unit disc. It follows from the work of Hoffman [8, p. 100], that $\Sigma(B)$ contains an element $\psi$ not on the Silov boundary such that $B_{\psi}=B_{\psi}^{2}$. (We are indebted to S. J. Sidney for bringing this to our attention.)

We verify first that if $f$ is in the closure of $C(\Gamma) \otimes P_{0}^{k}$ then $f \in \bigcap_{n=1}^{\infty} A_{\phi}^{n}$. This was shown by Sidney for elements of $C(\Gamma) \otimes P_{0}^{k}$. In general, $f=\lim f_{j}$ with $f_{j} \in C(\Gamma) \otimes P_{0}^{k}$. Fix $n$. Then $f_{j}=\sum_{i=k}^{n k-1} g_{i j} \otimes z^{i}+\sum h_{i} \otimes e_{i}$ with $e_{i} \in P_{0}^{n k}$. On each $\operatorname{disc}\{\gamma\} \times Z$ we have for each $i,\left|g_{i j}(\gamma)-g_{i m}(\gamma)\right| \leqq \max \left\{\left|f_{j}(\gamma, z)-f_{m}(\gamma, z)\right|: z \in Z\right\}$ $\leqq\left\|f_{j}-f_{m}\right\|$. Hence for each $i=k, \ldots, n k-1$, the sequence $\left\{g_{i j}\right\}$ converges to $g_{i}$. Now $f=\sum g_{i} \otimes z^{i}+h$, where $h$ is in the closure of $C(\Gamma) \otimes P_{0}^{n k}$, is in $A_{\phi}^{n}$ since this is true of each $g_{i} \otimes z^{i}$ and $h=\left(1 \otimes z^{k}\right)^{n-1} h^{\prime}$ for some $h^{\prime}$ in the closure of $C(\Gamma) \otimes P_{0}^{k}$.

We next assert that $\left\{1 \otimes z+A_{\phi}^{2}\right\}$ is a basis for $A_{\phi} / A_{\phi}^{2}$. We note first $1 \otimes z \notin A_{\Phi}^{2}$ since $1 \otimes z$ is orthogonal to $A_{\phi}^{2}$ in $L^{2}(\mu \times m)$ where $\mu$ is a representing measure on $\Gamma$ for $\psi$ and $m$ is normalized Lebesgue measure. On the other hand, an argument similar in spirit to that of the preceding paragraph yields that any $f \in A_{\phi}$ may be written $f=\alpha(1 \otimes z)+\sum_{j=0}^{k-1} h_{j} \otimes z^{j}+g$ where each $h_{j} \in B_{\psi}=B_{\psi}^{2}$ and $g$ is in the closure of $C(\Gamma) \otimes P_{0}^{k}$. Hence $1 \otimes z+A_{\phi}^{2}$ spans $A_{\phi} / A_{\phi}^{2}$.

By Theorem 2.3, $\operatorname{dim}\left(A_{\phi}^{j} / A_{\phi}^{j+1}\right)=0$ or 1 for each $j$. If $j \leqq k-1$, then $1 \otimes z^{j}$ is orthogonal to $A_{\phi}^{j+1}$ in $L^{2}(\mu \times m) .1 \otimes z^{k} \in C(\Gamma) \otimes P_{0}^{k} \subset \bigcap_{n=1}^{\infty} A_{\phi}^{n}$. Thus $\operatorname{dim}\left(A_{\phi}^{j} / A_{\phi}^{j+1}\right)$ $=1$ for $j \leqq k-1$ and $=0$ for $j \geqq k$ as asserted. It follows from Theorems 3.5 and 4.1 that $\phi$ is isolated in $\Sigma(A)$.

EXAMPLE 5.3. We close with an algebra whose spectrum contains a disc as an open subset in the Gelfand topology with the property that for $\phi$ in this disc, $\operatorname{dim}\left(A_{\phi} /\left(A_{\phi}^{2}\right)^{-}\right)$is not finite. We take $A$ to be an algebra of the same type as in the previous example, where now $I$ is an infinite index set and $B$ is the disc algebra. Then $\Sigma(A)=(D \times\{0\}) \cup(\Gamma \times Z)$ and $\phi=(0,0)$ is the center of an open analytic disc. On the other hand, if $m$ again denotes normalized Lebesgue measure on the unit circle and if $\lambda$ is the normalized Haar measure on $T$, then it is not difficult to 
verify that for each $i \in I, 1 \otimes z_{i}$ is orthogonal to $\left(A_{\phi}^{2}\right)^{-}$in $L^{2}(m \times \lambda)$. Hence $\left\{1 \otimes z_{i}+\left(A_{\phi}^{2}\right)^{-}: i \in I\right\}$ is an infinite linearly independent subset of $A_{\phi} /\left(A_{\phi}^{2}\right)^{-}$.

\section{BIBLIOGRAPHY}

1. A. Browder, Point derivations and analytic structure in the spectrum of a Banach algebra, J. Functional Analysis 7 (1971), 156-164.

2. P. C. Curtis, Jr., and A. Figá-Talamanca, Factorization theorems for Banach algebras, Proc. Internat. Sympos. on Function Algebras (Tulane Univ., 1965), Scott-Foresman, Chicago, Ill., 1966, pp. 169-185. MR 34 \#3350.

3. B. R. Gelbaum, Tensor products of Banach algebras, Canad. J. Math. 11 (1959), 297-310. MR 21 \#2922.

4. A. M. Gleason, Finitely generated ideals in Banach algebras, J. Math. Mech. 13 (1964), 125-132. MR 28 \#2458.

5. S. Goldberg, Unbounded linear operators, McGraw-Hill, New York, 1966. MR 34 \#580.

6. R. C. Gunning and H. Rossi, Analytic functions of several complex variables, PrenticeHall, Englewood Cliffs, N. J., 1965. MR 31 \#4927.

7. M. Hall, Jr., Combinatorial theory, Blaisdell, Waltham, Mass., 1967. MR 37 \#80.

8. K. Hoffman, Bounded analytic functions and Gleason parts, Ann. of Math. (2) 86 (1967), 74-111. MR 35 \#5945.

9. L. Hörmander, An introduction to complex analysis in several variables, Van Nostrand, Princeton, N. J., 1966. MR 34 \#2933.

10. S. J. Sidney, Properties of the sequence of closed powers of a maximal ideal in a sup-norm algebra, Trans. Amer. Math. Soc. 131 (1968), 128-148. MR 36 \#5701.

11. O. Zariski and P. Samuel, Commutative algebra. Vol. 2, University Series in Higher Math., Van Nostrand, Princeton, N. J., 1960. MR 22 \#11006.

Yale University, New Haven, Connecticut 06520

Western Washington State College, Bellingham, Washington 98225 